co-produce a tailored intervention framework for childhood asthma management by exploring the knowledge and attitudes towards asthma amongst WB and SA parents, carers and children.

Methods Semi-structured interviews with a purposive sample of 44 children aged 5-12yrs (33 SA, $14 \mathrm{WB}$ ) and 65 parents/carers (49 $\mathrm{SA}, 16 \mathrm{WB}$ ) were used to explore barriers and facilitators to asthma management. A comparative thematic analysis was conducted.

Results WB families were more likely to have pre-existing knowledge of asthma than SA families; previous knowledge of asthma strongly influenced how families managed childhood asthma in both communities. In a minority of SA families, 'fear of the unknown' prevented families from investigating asthma further. Beliefs regarding the causes and nature of asthma were similar in both groups, however whilst 33\% of SA families attributed asthma to either God's will or Karma, no WB families did so.

All communities reported that advice was often given by extended family members but this was more prominent in SA families, especially in relation to complementary asthma management strategies.

SA and WB families both reported a lack of information-giving by health care professionals in relation to asthma.

Conclusions Pre-existing knowledge and attitudes surrounding asthma differ between SA and WB parents and directly impact on management. Intervention Co-production is increasing in use and popularity. The MIA project supports the co-production model by highlighting the importance of identifying attitudes and beliefs towards asthma from different ethnic groups so that interventions can be tailored to address their fears and concerns more effectively. Disclaimer This project was funded by the National Institute for Health Research HS\&DR programme (ref 09/2001/19). The views and opinions expressed therein are those of the authors and do not necessarily reflect those of the HS\&DR programme, NIHR, NHS or the Department of Health.

\section{G121 ARE WE OVERINVESTIGATING HYPOGLYCAEMIA IN CHILDREN PRESENTING WITH GASTROENTERITIS IN A DISTRICT GENERAL HOSPITAL SETTING?}

doi:10.1136/archdischild-2013-304107.133
${ }^{1} \mathrm{~S}$ Austin, ${ }^{2} \mathrm{~S}$ Birch, ${ }^{2} \mathrm{~J}$ Walker, ${ }^{2} \mathrm{~N}$ Wickramsuriya, ${ }^{1} \mathrm{~V}$ Walker, ${ }^{3} \mathrm{~L}$ Wainwright, ${ }^{2} \mathrm{~S}$ Gray. 'Paediatrics, University Hospital Southampton, Southampton, UK; ${ }^{2}$ Paediatrics, Queen Alexandra Hospital, Portsmouth, UK; ${ }^{3}$ Biochemical Sciences, Queen Alexandra Hospital, Portsmouth, UK

Aims To develop a safe but less invasive approach to the investigation and management of hypoglycaemia in infants and children.

Methods 2010 We performed an audit of all hypoglycaemia screens undertaken between 1st February-12th May in a large district general hospital using retrospective case-note analysis.

Using these results we conducted a consultation exercise involving local consultants, biochemists and a tertiary metabolic specialist to develop a simple screening pathway that was also in line with the National Metabolic Biochemistry Network (NMBN) guidelines for investigation and management of hypoglycaemia. The pathway emphasises that symptomatic hypoglycaemia is a clinical emergency and permanent brain damage is a risk if treatment is delayed.

Table 1: 1st February 2011: The screening pathway was introduced into clinical practise

Table 2: 2011 \& 2012: Re-audit of clinical practise in both years for the same time period (1st February- 12th May)

Results In 2010, 22 hypoglycaemia screens were performed, all of which were normal, except for a recurrent finding of mildly elevated 3 hydroxy-butyrylcarnitine (as would be expected during fasting). 19 sets of case-notes were obtained. These showed 18/19 children had gastroenteritis, $17 / 19$ had no significant past medical history and 16/19 were admitted.

In 2011 and 2012 there was a substantial reduction in the number of screens performed in infants and children (10 in 2011; 7 in 2012). No significant abnormalities have been identified. The admission and follow up data for 2011 \& 2012 is currently being analysed.

Total numbers of infants and children referred to hospital, and the proportion of children diagnosed with gastroenteritis were similar in all three years.

Conclusions Analysis of practise in 2010 highlighted a population of previously well children who presented with symptoms of gastroenteritis and were incidentally found to have hypoglycaemia and were therefore investigated accordingly.

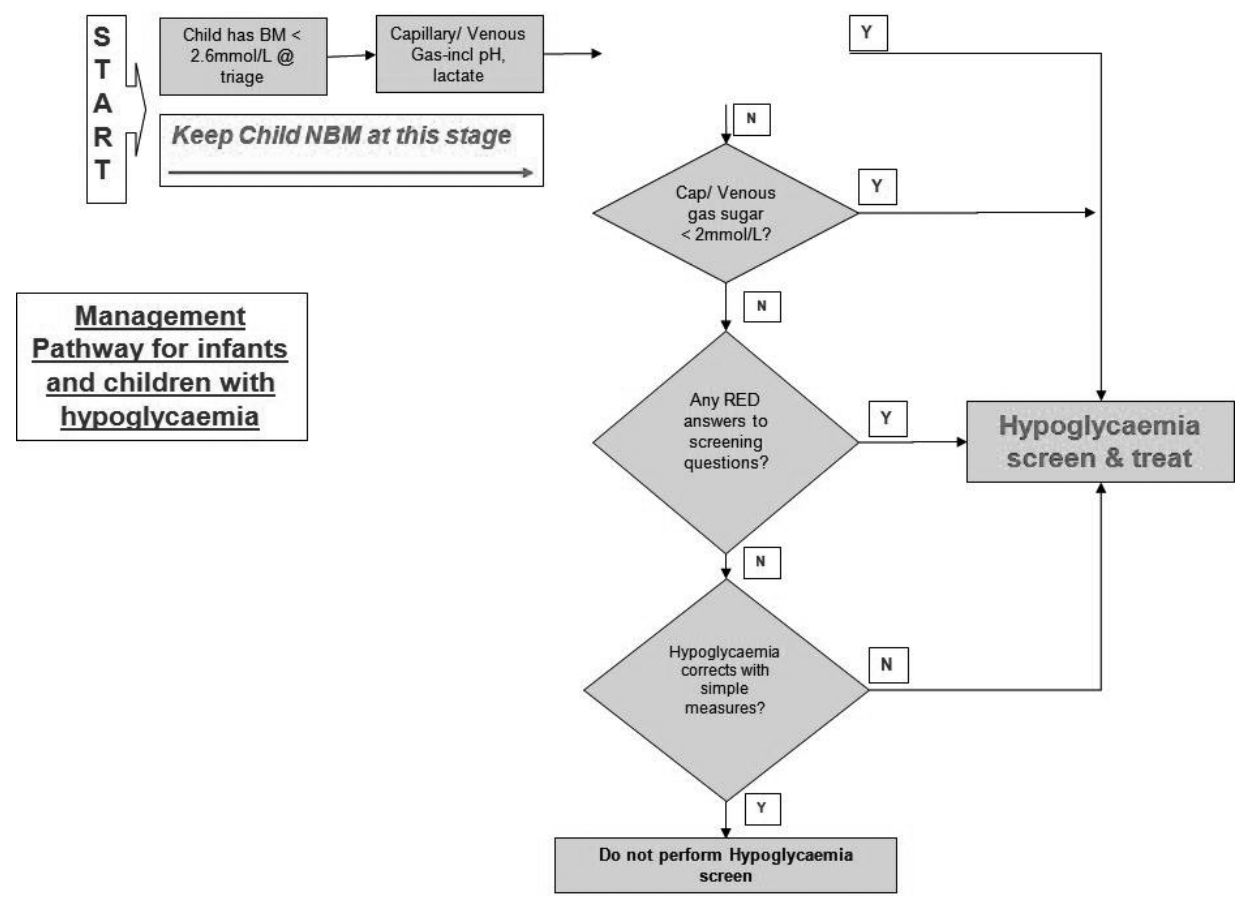




\begin{tabular}{|l|l|l|l|}
\hline & & YES & NO \\
\hline \hline & & YES & NO \\
\hline Presenting problem & Gastroenteritis type illness? & & \\
\hline Past Medical History & $\begin{array}{c}\text { Born after April 2009 and presumed normal } \\
\text { Guthrie? }\end{array}$ & Reassuring & \\
\hline & Normally well, no PMH of note? & & \\
\hline Focused History & Previous hx of low blood sugars? & & \\
\hline & Previous viral type illnesses with normal recovery? & & \\
\hline Can have period of fasting (e.g overnight) without \\
compromise?
\end{tabular}

\section{Abstract G121 Table 2}

We introduced a simple screening pathway for infants and children found to have hypoglycaemia. This approach remains in line with NMBN guidance.

It has resulted in $>50 \%$ reduction in hypoglycaemia screens performed. The cost savings are based upon reduced numbers of hypoglycaemia screens (£214), overnight admissions (£380/nt) and follow-up (£226).

The findings suggest that it may be possible to further refine our approach thereby reducing the number of hypoglycaemia screens performed without compromising patient safety.

\section{G122 SAFETY NETTING BEHAVIOUR OF PRIMARY HEALTHCARE PROFESSIONALS FOR ACUTELY SICK YOUNG CHILDREN: A QUALITATIVE STUDY}

doi:10.1136/archdischild-2013-304107.134

${ }^{1} \mathrm{CHD}$ Jones, ${ }^{2} \mathrm{~S}$ Neill, ${ }^{3} \mathrm{M}$ Lakhanpaul, ${ }^{4} \mathrm{D}$ Roland, ${ }^{1} \mathrm{M}$ Thompson,. ${ }^{\text {PPrimary }}$ Care Health Sciences, University of Oxford, Oxford, UK; ${ }^{2}$ School of Health, University of Northampton, Northampton, UK; ${ }^{3}$ General and Adolescent Paediatrics Unit, UCL Institute of Child Health, London, UK; ${ }^{4}$ Paediatric Emergency Medicine Leicester Academic Group, Leicester Royal Infirmary, Leicester, UK

Aims Acute illness is a frequent reason for consultation in primary care and an important cause of child morbidity and mortality. Healthcare professionals cannot correctly diagnose $100 \%$ of childhood illnesses at first consultation, so safety netting is used to extend the consultation and provide parents with information and resources to re-attend if necessary. UK childhood deaths from illnesses presenting to primary care exceed rates elsewhere in Europe, and safety netting has been introduced as a NICE quality standard for bacterial meningitis and meningococcal septicaemia; yet there is no standardised safety netting procedure. We aimed to explore the safety netting behaviour of frontline UK healthcare professionals for parents of acutely sick children under 5-years-old, including frequency, content, mode of delivery, and consistency.
Methods We conducted semi-structured focus groups and interviews with 16 doctors and nurses from general practise, emergency department and out-of-hours settings in the East Midlands, as part of the ASK SNIFF (Acutely Sick Kids, Safety Netting Intervention for Families) project. Data were analysed according to the grounded theory approach.

Results The content and delivery of safety netting was not consistent within or between organisations, whether it was written or verbal: "we've probably all got our favourite patient information leaflets that we give... not at the moment standardised across the practise" (GP surgery doctor); "I know what I say but you know, do we all say the same thing?" (Paediatric ED doctor). Factors influencing safety netting provision included perceived parental anxiety and confidence, healthcare professional parental status and experience, and time. Participants highlighted difficulty in knowing how often safety netting occurs, whether it is understood by parents, and its effectiveness: "often they'll nod their heads and say yes I understand everything you say and walk off and they might have no idea what we've just said" (Regular ED nurse). Other limitations were the broad, nonspecific nature of childhood illnesses, and parental difficulty interpreting information.

Conclusion Healthcare professionals lack standardised methods of safety netting. Addressing this gap in the management of acutely sick children may have potential to improve the efficiency of acute children's services, and reduce avoidable morbidity and mortality.

\section{G123 USE OF NON-PRESCRIBED MEDICINES, SUPPLEMENTS AND THERAPIES BY CHILDREN WITH A CHRONIC ILLNESS AND CHILDREN WITH AN ACUTE INJURY - A COMPARATIVE MIXED-METHODS STUDY IN UK}

doi:10.1136/archdischild-2013-304107.135

${ }^{1} \mathrm{M}$ Hurley, ${ }^{2} \mathrm{~J}$ Gates, ${ }^{2} \mathrm{~J} \mathrm{Ho},{ }^{3} \mathrm{~A}$ Gaunt, ${ }^{1} \mathrm{~A}$ Smyth. ${ }^{1}$ Academic Child Health, University of Nottingham, Nottingham, UK; ${ }^{2}$ Medical School, University of Nottingham, Nottingham, UK; ${ }^{3}$ Education Development \& Research, Warwick Medical School, Warwick, UK 